\title{
OPERATORS FROM BANACH SPACES TO COMPLEX INTERPOLATION SPACES
}

\author{
VERNON WILLIAMS
}

Abstract. Given a closed linear operator $A$ with dense domain in a Banach space $X, M$. Schechter [4] utilized the Lebesgue integral to construct a family of bounded linear operators from $X$ to the Calderón complex interpolation space $(X, D(A)),[2]$, where $D(A)$, the domain of $A$ in $X$, is a Banach space under the norm

$$
\|x\|_{D(A)}=\|x\|+\|A x\| .
$$

In this paper we utilize the complex functional calculus, which provides a more natural setting, to construct a similar family of operators. At the same time we achieve a strengthening of the Schechter result, for in the proof of our theorem we make no use of the adjoint $A^{*}$ of $A$ and consequently do not require the domain of $A$ to be dense in $X$. A completely analogous procedure would permit the removal from the Schechter theorem, referred to above, of the hypothesis that the domain of $A$ is dense in $X$.

Given a closed linear operator $A$ with dense domain in a Banach space $X, M$. Schechter [4] utilized the Lebesgue integral to construct a family of bounded linear operators from $X$ to the Calderón complex interpolation space $(X, D(A))$, [2], where $D(A)$, the domain of $A$ in $X$, is a Banach space under the norm

$$
\|x\|_{D(A)}=\|x\|+\|A x\| \text {. }
$$

In this paper we utilize the complex functional calculus, which provides a more natural setting, to construct a similar family of operators. At the same time we achieve a strengthening of the Schechter result, for in the proof of our theorem we make no use of the adjoint $A^{*}$ of $A$ and consequently do not require the domain of $A$ to be dense in $X$. A completely analogous procedure would permit the removal from the Schechter theorem referred to above of the hypothesis that the domain of $A$ is dense in $X$.

Theorem. (a) Let $X$ be a complex Banach space. Let $A$ be a closed linear operator in $X$ such that the resolvent set of $A$ is nonempty, and the spectrum of $A$ is contained in a Cauchy domain; that is, a subset $D$ of the complex plane with the following properties:

Received by the editors October 18, 1969.

AMS 1969 subject classifications. Primary 4725, 4638; Secondary 4730.

Key words and phrases. Calderón complex interpolation space, functional calculus, closed linear operator with spectrum contained in a Cauchy domain. 
(1) $D$ is open.

(2) D has a finite number of components, the closures of any two of which are disjoint.

(3) The boundary $B(D)$ of $D$ is composed of a finite number of closed rectifiable Jordan curves, no two of which intersect.

(b) Let $\phi(w, z)$ be a complex-valued function defined for each $w$ in the closure of the open strip $S$ in the complex plane between 0 and 1, and for each $z$ in a set $N$ containing a neighborhood of the closure of the Cauchy domain D, together with a neighborhood of infinity. Suppose further that $\phi(w, z)$ satisfies the following:

(1) There exists a constant $C$ such that for $w_{1}, w_{2}$ in the closure of the open strip $S$, and $z$ in $B(D)$, then

$$
\left|\phi\left(w_{1}, z\right)-\phi\left(w_{2}, z\right)\right| \leqq C\left|w_{1}-w_{2}\right| .
$$

(2) Let $w$ in the closure of $S$ be fixed, then $\phi(w, z)$ is an analytic function of $z$ in a neighborhood of the closure of $D$ and at infinity.

(3) For each $w$ in $S$, and each $z$ in $B(D)$, the partial derivative $\phi_{w}(w, z)$ exists and is continuous in $z$ on $B(D)$. We assume further that

$$
\left|(\phi(w+h, z)-\phi(w, z)) / h-\phi_{w}(w, z)\right|
$$

converges to 0 uniformly in $\mathrm{z}$ on $B(D)$ as $h$ approaches 0.

(4) For $j=0,1$, there exist constants $K_{j}$ such that for $t$ real and $z$ in $B(D)$,

$$
|\phi(j+i t, z)| \leqq K_{j} .
$$

(c) $B(D)$ is rectifiable by assumption (a). Let $L$ denote the length of $B(D)$. Let $M$ denote the maximum value achieved by the continuous function $\left\|(z-A)^{-1}\right\|$ on the compact set $B(D)$. Let $s$ satisfying $0<s<1$ be fixed. $B(D)$ is compact. Let $T$ denote the maximum of $\{|z| \mid z$ is a member of $B(D)\}$.

Then $\phi(w, z)$ induces a bounded linear mapping $\Phi_{s}(A)$ from $X$ to the Calderón complex interpolation space $(X, D(A))_{8}$ with norm $\leqq \max \left\{K_{0} M L, K_{1} L(1+M+T M)\right\}$.

REMARK. Observe that in particular an arbitrary constant function $\phi(w, z)$ satisfies the four conditions in assumption (b). We note further that $\phi(w, z)$ satisfies all conditions in assumption (b) if $\phi(w, z)=f(w) g(z)$ where $f(w)$ is such that:

(1) $f(w)$ is analytic in the open strip $S$.

(2) $f(w)$ satisfies a Lipschitz condition in the closure of $S$.

(3) There exist constants $K_{j}, j=0,1$, such that $|f(j+i t)| \leqq K_{j}$ for all real $t$. 
(For example $f(w)=1 /(w+1)^{n}, n$ a positive integer, and $g(z)$ is analytic in a neighborhood of the closure of $D$, and at infinity.) (For example $g(z)=1 /(z-a)^{n}$, where $n$ is a positive integer and " $a$ " is any point of the resolvent set of $A$ which does not belong to the closure of the Cauchy domain $D$.)

Proof of Theorem. We first recall the definition of the Calderón interpolation space $(X, D(A))$. [1]

(1) $X+D(A)=\left\{x_{0}+x_{1} \mid x_{0} \in X\right.$ and $\left.x_{1} \in D(A)\right\}$ is a Banach space under the norm

$$
\|x\|_{X+D(\Lambda)}=\inf _{x_{0}+x_{1}=x}\left\{\left\|x_{0}\right\|_{X}+\left\|x_{1}\right\|_{D(A)}\right\} .
$$

(2) Let $H(X, D(A))$ be the set of all functions $f$ from the closure of the open strip $S$ to $X+D(A)$ which satisfy:

(i) $f$ is analytic in $S$.

(ii) $f$ is continuous on the closure of $S$.

(iii) For each real $t, f(i t) \in X, f(1+i t) \in D(A)$ and there exist constants $C_{0}, C_{1}$ such that for all real $t\|f(i t)\|_{X} \leqq C_{0}$ and $\|f(1+i t)\|_{D(A)}$ $\leqq C_{1}$.

$H(X, D(A))$ is a Banach space under the norm

$$
\|f\|_{H(X, D(\boldsymbol{A}))}=\max \left\{\sup _{t}\|f(i t)\|_{X}, \sup _{t}\|f(1+i t)\|_{D(A)}\right\} .
$$

(3) $(X, D(A))_{s}=\{x \mid x=f(s), f \in H(X, D(A))\}$ is a Banach space under the norm $\|x\|_{(X, D(A)) s}=\inf _{f(s)=x}\left\{\|f\|_{H(X, D(A))}\right\}$.

We show in the sequel that each of the following is valid:

(I) For $w$ fixed in the closure of $S$,

$$
\phi(w, A)=\int_{B(D)} \phi(w, z)(z-A)^{-1} d z
$$

is a bounded linear operator mapping $X$ into itself.

(II) For $x$ fixed in $X, \phi(w, A) x$ is a continuous mapping of the closure of $S$ into $X$.

(III) For $x$ fixed in $X, \phi(w, A) x$ is an analytic mapping of $S$ into $X$.

(IV) For $x$ fixed in $X, \phi(w, A) x$ is a function in $H(X, D(A))$ satisfying

$$
\|\phi(w, A) x\|_{H(X, D(\mathcal{A}))} \leqq \max \left\{K_{0} M L, K_{1} L(1+M+T M)\right\}\|x\| .
$$

Assuming for the time being I, II, III and IV above, it is clear that mapping $\Phi_{\imath}(A)$ of $X$ into $(X, D(A))_{\text {s }}$ defined by

$$
\Phi_{\imath}(A) x=[\phi(w, A) x](s)=\phi(s, A) x
$$


is the required bounded operator of our theorem since

(1) For $x_{1}, x_{2}$ in $X$ and $a_{1}, a_{2}$ scalars,

$$
\begin{aligned}
\Phi_{s}(A)\left(a_{1} x_{1}+a_{2} x_{2}\right) & =\left[\phi(w, A)\left(a_{1} x_{1}+a_{2} x_{2}\right)\right](s) \\
& =\phi(s, A)\left(a_{1} x_{1}+a_{2} x_{2}\right) \\
& =a_{1} \phi(s, A) x_{1}+a_{2} \phi(s, A) x_{2} \\
& =a_{1} \Phi_{s}(A) x_{1}+a_{2} \Phi_{s}(A) x_{2} .
\end{aligned}
$$

Thus, the operator $\Phi_{a}(A)$ is linear.

(2) For $x$ in $X$,

$$
\begin{aligned}
\left\|\Phi_{s}(A) x\right\|_{(X, D(A)),} & \inf _{f(s)=\Phi_{g}(A) x}\left\{\|f\|_{H(X, D(A))}\right\} \\
& \leqq\|\phi(w, A) x\|_{H(X, D(A))} \\
& \leqq \max \left\{K_{0} M L, K_{1} L(1+M+T M)\right\}\|x\| .
\end{aligned}
$$

Hence, $\Phi_{\varepsilon}(A)$ is bounded with norm satisfying

$$
\left\|\Phi_{s}(A)\right\| \leqq \max \left\{K_{0} M L, K_{1} L(1+M+T M)\right\} .
$$

We proceed now to establish the validity of I, II, III and IV.

(1) Statement (I) is a direct consequence of assumptions (a), (b), part (2), and a classical result of complex functional analysis [3].

(2) To verify (II), we recall that $B(D)$, the boundary of $D$, is rectifiable with length $L$ and that $\left\|(z-A)^{-1}\right\|$ is continuous on $B(D)$ with maximum value $M$. Let $x$ in $X$ be fixed. Let $w$, $w_{0}$ be points of the closure of the open strip $S$. Then

$$
\begin{aligned}
& \left\|\phi(w, A) x-\phi\left(w_{0}, A\right) x\right\| \\
& \quad=\left\|\left(\int_{B(D)} \phi(w, z)(z-A)^{-1} d z\right) x-\left(\int_{B(D)} \phi\left(w_{0}, z\right)(z-A)^{-1} d z\right) x\right\| \\
& \quad=\left\|\left(\int_{B(D)}\left(\phi(w, z)-\phi\left(w_{0}, z\right)\right)(z-A)^{-1} d z\right) x\right\| \\
& \quad \leqq\left\{\int_{B(D)}\left|\phi(w, z)-\phi\left(w_{0}, z\right)\right|\left\|(z-A)^{-1}\right\||d z|\right\}\|x\| \\
& \quad \leqq C\left|w-w_{0}\right|\left(\int_{B(D)}\left\|(z-A)^{-1}\right\||d z|\right)\|x\|
\end{aligned}
$$

(by the Lipschitz condition of assumption (b))

$$
\leqq C M L\left|w-w_{0}\right|\|x\| \text {. }
$$

The continuity of $\phi(w, A) x$ on the closure of $S$ is now evident. 
(3) We establish (III) as follows. In accordance with assumption (b), part (3), the partial derivative $\phi_{w}(w, z)$ exists for $w$ in $S$ and $z$ in $B(D)$, and is continuous in $z$ on $B(D)$ for each fixed $w$ in $S$. Therefore, $\int_{B(D)} \phi_{w}(w, z)(z-A)^{-1} d z$ exists.

Now let a positive number $\epsilon$ be given. Then for complex $h$ suft ciently small in absolute value one has

$$
\begin{aligned}
& \left\|(\phi(w+h, A) x-\phi(w, A) x) / h-\left(\int_{B(D)} \phi_{w}(w, z)(z-A)^{-1} d z\right) x\right\| \\
& \quad=\left\|\left\{\int_{B(D)}\left\{(\phi(w+h, z)-\phi(w, z)) / h-\phi_{w}(w, z)\right\}(z-A)^{-1} d z\right\} x\right\| \\
& \quad \leqq\left\{\int_{B(D)}\left|(\phi(w+h, z)-\phi(w, z)) / h-\phi_{w}(w, z)\right|\left\|(z-A)^{-1}\right\||d z|\right\}\|x\| \\
& \quad \leqq\left\{\int_{B(D)} \epsilon\left\|(z-A)^{-1}\right\||d z|\right\}\|x\| \quad \text { (by assumption (b), part (3)) } \\
& \leqq \epsilon M L\|x\| .
\end{aligned}
$$

The analyticity of $\phi(w, A) x$ in $S$ is now clear.

(4) Given the previous results concerning the continuity and analyticity of $\phi(w, A) x$, one is able to show that $\phi(w, A) x$ is a member of $H(X, D(A))$ by ascertaining the following:

(i) For $t$ real, $\phi(1+i t, A) x$ is a member of $D(A)$.

(ii) There exist constants $M_{0}, M_{1}$ such that

$$
\begin{aligned}
& \|\phi(1+i t, A) x\|_{D(A)} \leqq M_{1} \text { for all real } t, \text { and } \\
& \|\phi(i t, A) x\| \leqq M_{0} \text { for all real } t \text {. }
\end{aligned}
$$

Let $t$ be a fixed real number and let $x$ be a fixed member of $X$. Let $y=\phi(1+i t, A) x$. Then $y=\left(\int_{B(D)} \phi(1+i t, z)(z-A)^{-1} d z\right) x$. Thus there exists a sequence of partitions $P_{n}$ of $B(D)$ with "norms" converging to 0 , and a corresponding sequence of "Riemann sums",

$$
S_{n}=\sum_{k=1}^{n} \phi\left(1+i t, z_{k}^{\prime}\right)\left(z_{k}^{\prime}-A\right)^{-1}\left(z_{k}-z_{k-1}\right)
$$

satisfying $S_{n} x$ converges to $y$ as $n$ approaches infinity.

One observes that for each $x$ in $X, S_{n} x$ is an element of $D(A)$. The operator $A$ by assumption is closed in $X$. Thus, to establish that $y$ is a member of $D(A)$, it is sufficient to show that the sequence $A\left(S_{n} x\right)$ has a limit as $n$ approaches infinity. We presently obtain this 
result. Recall that $B(D)$, the boundary of $D$ is compact. Thus the set,

$$
\{|z| \mid z \text { is a member of } B(D) \text {, }
$$

has a maximum value which we denote by " $T$ ". Now let $z \in B(D)$. Then

$$
\begin{aligned}
\left\|A(z-A)^{-1}\right\| & =\left\|((A-z)+z)(z-A)^{-1}\right\| \\
& \leqq\left\|(A-z)(z-A)^{-1}\right\|+|z|\left\|(z-A)^{-1}\right\| \\
& =\|I\|+|z|\left\|(z-A)^{-1}\right\| \quad(I \text { is the identity operator }) \\
& \leqq 1+T M .
\end{aligned}
$$

Thus, for each $z$ in $B(D), A(z-A)^{-1}$ is a bounded linear operator on $X$. Furthermore, $A(z-A)^{-1}$ is continuous on $B(D)$, for let $z, z_{0}$ be members of $B(D)$. Then

$$
\begin{aligned}
\left\|A(z-A)^{-1}-A\left(z_{0}-A\right)^{-1}\right\| & =\left\|A\left((z-A)^{-1}-\left(z_{0}-A\right)^{-1}\right)\right\| \\
& =\left\|A\left(z_{0}-z\right)(z-A)^{-1}\left(z_{0}-A\right)^{-1}\right\| \\
& \leqq\left|z_{0}-z\right|\left\|A(z-A)^{-1}\right\|\left\|\left(z_{0}-A\right)^{-1}\right\| \\
& \leqq\left|z_{0}-z\right|(1+T M) M .
\end{aligned}
$$

By assumption (b), part (2) $\phi(1+i t, z)$ is an analytic function of $z$ in a neighborhood of the closure of $D$. Hence, it is certainly continuous on $B(D)$. Thus $\int_{B(D)} \phi(1+i t, z) A(z-A)^{-1} d z$ exists. Consequently,

$$
A\left(S_{n} x\right)=\left\{\sum_{k=1}^{n} \phi\left(1+i t, z_{k}^{\prime}\right) A\left(z_{k}^{\prime}-A\right)^{-1}\left(z_{k}-z_{k-1}\right)\right\} x
$$

converges to $\left\{\int_{B(D)} \phi(1+i t, z) A(z-A)^{-1} d z\right\} x$ as $n$ approaches infinity. Thus, $y \in D(A)$.

One now observes that

$$
\begin{aligned}
\|\phi(1+i t, A) x\| & =\left\|\left\{\int_{B(D)} \phi(1+i t, z)(z-A)^{-1} d z\right\} x\right\| \\
& \leqq\left\{\int_{B(D)}|\phi(1+i t, z)|\left\|(z-A)^{-1}\right\||d z|\right\}\|x\| \\
& \leqq\left\{\int_{B(D)} K_{1}\left\|(z-A)^{-1}\right\||d z|\right\}\|x\|
\end{aligned}
$$

(by assumption (b), part (4))

$\leqq K_{1} M L\|x\|$ 
Thus,

$$
\begin{aligned}
\|\phi(1+i t, A) x\|_{D(A)}= & \|\phi(1+i t, A) x\|+\|A(\phi(1+i t, A) x)\| \\
= & \left\|\left\{\int_{B(D)} \phi(1+i t, z)(z-A)^{-1} d z\right\} x\right\| \\
& +\left\|\left\{\int_{B(D)} \phi(1+i t, z) A(z-A)^{-1} d z\right\} x\right\| \\
\leqq & K_{1} M L\|x\|+K_{1}(1+T M) L\|x\| \\
= & \left\{K_{1} M L+K_{1}(1+T M) L\right\}\|x\| \\
= & \left\{K_{1} L(1+M+T M)\right\}\|x\| .
\end{aligned}
$$

Similarly,

$$
\begin{aligned}
\|\phi(i t, A) x\| & =\left\|\left\{\int_{B(D)} \phi(i t, z)(z-A)^{-1} d z\right\} x\right\| \\
& \leqq\left\{\int_{B(D)}|\phi(i t, z)|\left\|(z-A)^{-1}\right\||d z|\right\}\|x\| \\
& \leqq\left\{\int_{B(D)} K_{0}\left\|(z-A)^{-1}\right\||d z|\right\}\|x\|
\end{aligned}
$$

(by assumption (b), part (4))

$$
\leqq K_{0} M L\|x\| \text {. }
$$

Thus, $\phi(w, A) x$ is a member of $H(X, D(A))$, and

$$
\begin{aligned}
\|\phi(w, A) x\|_{H(X, D(A))}= & \max \left\{\sup _{t}\|\phi(i t, A) x\|, \sup _{t}\|\phi(1+i t, A) x\|_{D(A)}\right\} \\
& \leqq \max \left\{\left(K_{0} M L\right)\|x\|, K_{1} L(1+M+T M)\|x\|\right\} \\
& =\max \left\{K_{0} M L, K_{1} L(1+M+T M)\right\}\|x\| .
\end{aligned}
$$

\section{BIBLIOGRAPHY}

1. A. P. Calderon, Intermediate spaces and interpolation, Studia Math. Special Series 1 (1963), 31-34. MR 26 \#5409.

2. - Intermediate spaces and interpolation, the complex method, Studia Math. 24 (1964), 113-190. MR 29 \#5097.

3. N. Dunford and J. T. Schwartz, Linear operators. I: General theory, Pure and Appl. Math., vol. 7, Interscience, New York, 1958, pp. 601-602. MR 22 \#8302.

4. M. Schechter, Complex interpolation, Compositio Math. 18 (1967), 117-147. MR 36 \#6927.

Rutgers University, New Brunswick, New Jersey 08903 